\title{
Algorithms with variant anchors for pseudocontractive mappings
}

\author{
Lijin Guo ${ }^{1}$, Shin Min Kang ${ }^{2 *}$ and Chahn Yong Jung ${ }^{3 *}$
}

\section{"Correspondence:}

smkang@gnu.ac.kr;

bb5734@gnu.ac.kr

${ }^{2}$ Department of Mathematics and

RINS, Gyeongsang National University, Jinju, 660-701, Korea

${ }^{3}$ Department of Business

Administration, Gyeongsang

National University, Jinju, 660-701,

Korea

Full list of author information is

available at the end of the article

\begin{abstract}
The purpose of this paper is to find the fixed points of pseudocontractive mappings by using the iterative technique. Two algorithms with variant anchors have been introduced. Strong convergence results are given. Especially, we can find the minimum-norm fixed point of pseudocontractive mappings.
\end{abstract}

MSC: $47 \mathrm{H} 05 ; 47 \mathrm{H} 10 ; 47 \mathrm{H} 17$

Keywords: algorithms; pseudocontractive mapping; fixed point; strong convergence

\section{Introduction}

In this paper, we assume that $H$ is a real Hilbert space and $C \subset H$ is a nonempty closed convex subset. Recall that a mapping $T: C \rightarrow C$ is said to be Lipschitzian if

$$
\left\|T u-T u^{\dagger}\right\| \leq \kappa\left\|u-u^{\dagger}\right\|, \quad \forall u, u^{\dagger} \in C,
$$

where $\kappa>0$ is a constant, which is in general called the Lipschitz constant. If $\kappa=1, T$ is called nonexpansive.

A mapping $T: C \rightarrow C$ is said to be pseudocontractive if

$$
\left\langle T u-T u^{\dagger}, u-u^{\dagger}\right\rangle \leq\left\|u-u^{\dagger}\right\|^{2}, \quad \forall u, u^{\dagger} \in C .
$$

We use Fix $(T)$ to denote the set of fixed points of $T$.

In the literature, there are a large number references associated with the fixed point algorithms for the pseudocontractive mappings. See, for instance, [1-24]. (The interest of pseudocontractions lies in their connection with monotone operators; namely, $T$ is a pseudocontraction if and only if the complement $I-T$ is a monotone operator.)

Now there exists an example which shows that Mann iteration does not converge for the pseudocontractive mappings [2]. At present, it is still an interesting topic to construct algorithms for finding the fixed points of the pseudocontractive mappings.

On the other hand, there are perturbations always occurring in the iterative processes because the manipulations are inaccurate. Recently, in order to find the fixed points of the nonexpansive mappings, Yao and Shahzad [25] introduced the following algorithms with perturbations and obtained the strong convergence results.

(c) 2014 Guo et al: licensee Springer. This is an Open Access article distributed under the terms of the Creative Commons Attribution License (http://creativecommons.org/licenses/by/2.0), which permits unrestricted use, distribution, and reproduction in any medium, provided the original work is properly cited. 
Algorithm 1.1 Let $C$ be a nonempty closed convex subset of a real Hilbert space $H$. Let $T: C \rightarrow C$ be a nonexpansive mapping. For given $x_{0} \in C$, define a sequence $\left\{x_{m}\right\}$ in the following manner:

$$
x_{m}=\operatorname{proj}_{C}\left[\alpha_{m} u_{m}+\left(1-\alpha_{m}\right) T x_{m}\right], \quad m \geq 0,
$$

where $\left\{\alpha_{m}\right\}$ is a sequence in $[0,1]$ and the sequence $\left\{u_{m}\right\} \subset H$ is a small perturbation for the $m$-step iteration satisfying $\left\|u_{m}\right\| \rightarrow 0$ as $m \rightarrow \infty$.

Theorem 1.2 Suppose $\operatorname{Fix}(T) \neq \emptyset$. Then, as $\alpha_{m} \rightarrow 0$, the sequence $\left\{x_{m}\right\}$ generated by the implicit method (1.1) converges to $\tilde{x} \in \operatorname{Fix}(T)$, which is the minimum-norm fixed point of $T$.

Algorithm 1.3 Let $C$ be a nonempty closed convex subset of a real Hilbert space $H$. Let $T: C \rightarrow C$ be a nonexpansive mapping. For given $x_{0} \in C$, define a sequence $\left\{x_{n}\right\}$ in the following manner:

$$
x_{n+1}=\left(1-\beta_{n}\right) x_{n}+\beta_{n} \operatorname{proj}_{C}\left[\alpha_{n} u_{n}+\left(1-\alpha_{n}\right) T x_{n}\right], \quad n \geq 0,
$$

where $\left\{\alpha_{n}\right\}$ and $\left\{\beta_{n}\right\}$ are two sequences in $(0,1)$ and the sequence $\left\{u_{n}\right\} \subset H$ is a perturbation for the $n$-step iteration.

Theorem 1.4 Suppose that $\operatorname{Fix}(T) \neq \emptyset$. Assume the following conditions are satisfied:

(i) $\lim _{n \rightarrow \infty} \alpha_{n}=0$ and $\sum_{n=0}^{\infty} \alpha_{n}=\infty$;

(ii) $0<\liminf _{n \rightarrow \infty} \beta_{n} \leq \limsup _{n \rightarrow \infty} \beta_{n}<1$;

(iii) $\sum_{n=0}^{\infty} \alpha_{n}\left\|u_{n}\right\|<\infty$.

Then the sequence $\left\{x_{n}\right\}$ generated by the explicit iterative method (1.2) converges to $\tilde{x} \in$ $\operatorname{Fix}(T)$, which is the minimum-norm fixed point of $T$.

Note that the idea of the iterative algorithms with perturbations has been extended to the other topics, see, for example, [26].

Motivated by the above ideas and the results in the literature, in the present paper, we present two algorithms with variant anchors for finding the fixed points of the pseudocontractive mappings in Hilbert spaces. Strong convergence results are given. As special cases, we can find the minimum-norm fixed point of the pseudocontractive mappings.

\section{Preliminaries}

Recall that the metric projection $\operatorname{proj}_{C}: H \rightarrow C$ is defined by

$$
\operatorname{proj}_{C} x:=\arg \min _{\forall y \in C}\|x-y\|, \quad x \in H .
$$

It is obvious that $\operatorname{proj}_{C}$ satisfies

$$
\left\|x-\operatorname{proj}_{C} x\right\| \leq\|x-y\|, \quad \forall y \in C,
$$

and is characterized by

$$
\operatorname{proj}_{C} x \in C, \quad\left\langle x-\operatorname{proj}_{C} x, y-\operatorname{proj}_{C} x\right\rangle \leq 0, \quad \forall y \in C .
$$

The following two lemmas will be useful for our main results. 
Lemma 2.1 ([24]) Let $C$ be a closed convex subset of a Hilbert space H. Let $T: C \rightarrow C$ be a Lipschitzian pseudocontractive mapping. Then $\operatorname{Fix}(T)$ is a closed convex subset of $C$ and the mapping $I-T$ is demiclosed at 0 , i.e., whenever $\left\{x_{n}\right\} \subset C$ is such that $x_{n} \rightarrow x$ and $(I-T) x_{n} \rightarrow 0$, then $(I-T) x=0$.

Lemma 2.2 ([27]) Assume $\left\{a_{n}\right\}$ is a sequence of nonnegative real numbers such that

$$
a_{n+1} \leq\left(1-\gamma_{n}\right) a_{n}+\gamma_{n} \delta_{n}, \quad n \geq 0
$$

where $\left\{\gamma_{n}\right\}$ is a sequence in $(0,1)$ and $\left\{\delta_{n}\right\}$ is a sequence in $R$ such that

(i) $\sum_{n=0}^{\infty} \gamma_{n}=\infty$;

(ii) $\lim \sup _{n \rightarrow \infty} \delta_{n} \leq 0$ or $\sum_{n=0}^{\infty}\left|\delta_{n} \gamma_{n}\right|<\infty$.

Then $\lim _{n \rightarrow \infty} a_{n}=0$.

\section{Main results}

In the sequel, we assume that $C$ is a nonempty closed convex subset of a real Hilbert space $H$ and $T: C \rightarrow C$ is a $\kappa$-Lipschitzian pseudocontractive mapping with nonempty fixed points set $\operatorname{Fix}(T)$.

The first result is on the convergence of the path for the pseudocontractive mappings. Now, we define our path as follows.

For fixed $\zeta, t \in(0,1)$ and $u_{t} \in H$, we define a mapping $G_{t}: C \rightarrow C$ by

$$
G_{t} x=(1-\zeta) \operatorname{proj}_{C}\left[t u_{t}+(1-t) x\right]+\zeta T x, \quad \forall x \in C,
$$

where $\operatorname{proj}_{C}: H \rightarrow C$ is the metric projection from $H$ on $C$.

Next, we show that the mapping $G_{t}$ is strongly pseudocontractive. Indeed, for $x, y \in C$, we have

$$
\begin{aligned}
\left\langle G_{t} x-G_{t} y, x-y\right\rangle= & (1-\zeta)\left\langle\operatorname{proj}_{C}\left[t u_{t}+(1-t) x\right]-\operatorname{proj}_{C}\left[t u_{t}+(1-t) y\right], x-y\right\rangle \\
& +\zeta\langle T x-T y, x-y\rangle \\
\leq & (1-\zeta)\left\|\operatorname{proj}_{C}\left[t u_{t}+(1-t) x\right]-\operatorname{proj}_{C}\left[t u_{t}+(1-t) y\right]\right\|\|x-y\| \\
& +\zeta\|x-y\|^{2} \\
\leq & (1-\zeta)(1-t)\|x-y\|^{2}+\zeta\|x-y\|^{2} \\
= & {[1-(1-\zeta) t]\|x-y\|^{2} . }
\end{aligned}
$$

Since $\zeta, t \in(0,1), 1-(1-\zeta) t \in(0,1)$. Hence, $G_{t}$ is a strongly pseudocontractive mapping. By [2], $G_{t}$ has a unique fixed point $x_{t} \in C$. That is, $x_{t}$ satisfies

$$
x_{t}=(1-\zeta) \operatorname{proj}_{C}\left[t u_{t}+(1-t) x_{t}\right]+\zeta T x_{t}, \quad \forall t \in(0,1) .
$$

Remark 3.1 $u_{t} \in H$ can be seen as a perturbation.

Next, we prove the convergence of the path (3.1).

Theorem 3.2 If $\lim _{t \rightarrow 0} u_{t}=u \in H$, then the path $\left\{x_{t}\right\}$ defined by (3.1) converges strongly to $\operatorname{proj}_{\mathrm{Fix}(T)}(u)$. 
Proof Let $p \in \operatorname{Fix}(T)$. We get from (3.1) that

$$
\begin{aligned}
\left\|x_{t}-p\right\|^{2} & =(1-\zeta)\left\langle\operatorname{proj}_{C}\left[t u_{t}+(1-t) x_{t}\right]-p, x_{t}-p\right\rangle+\zeta\left\langle T x_{t}-p, x_{t}-p\right\rangle \\
& \leq(1-\zeta)\left\|\operatorname{proj}_{C}\left[t u_{t}+(1-t) x_{t}\right]-p\right\|\left\|x_{t}-p\right\|+\zeta\left\|x_{t}-p\right\|^{2} \\
& \leq(1-\zeta)\left\|t\left(u_{t}-p\right)+(1-t)\left(x_{t}-p\right)\right\|\left\|x_{t}-p\right\|+\zeta\left\|x_{t}-p\right\|^{2} \\
& \leq(1-\zeta)\left[(1-t)\left\|x_{t}-p\right\|+t\left\|u_{t}-p\right\|\right]\left\|x_{t}-p\right\|+\zeta\left\|x_{t}-p\right\|^{2}
\end{aligned}
$$

It follows that

$$
\left\|x_{t}-p\right\| \leq\left\|u_{t}-p\right\|
$$

Since $\lim _{t \rightarrow 0} u_{t}=u \in H$, there exists a constant $M>0$ such that $\sup _{t \in(0,1)}\left\|u_{t}-u\right\| \leq M$. So,

$$
\left\|x_{t}-p\right\| \leq\left\|u_{t}-p\right\| \leq\left\|u_{t}-u\right\|+\|u-p\| \leq M+\|u-p\| .
$$

Thus, $\left\{x_{t}\right\}$ is bounded.

By (3.1), we have

$$
\begin{aligned}
\left\|x_{t}-T x_{t}\right\| & =\left\|(1-\zeta) \operatorname{proj}_{C}\left[t u_{t}+(1-t) x_{t}\right]+\zeta T x_{t}-T x_{t}\right\| \\
& \leq(1-\zeta)\left\|\operatorname{proj}_{C}\left[t u_{t}+(1-t) x_{t}\right]-T x_{t}\right\| \\
& \leq(1-\zeta)\left[\left\|x_{t}-T x_{t}\right\|+t\left\|u_{t}-x_{t}\right\|\right] .
\end{aligned}
$$

Therefore,

$$
\begin{aligned}
& \left\|x_{t}-T x_{t}\right\| \leq \frac{(1-\zeta) t}{\zeta}\left\|u_{t}-x_{t}\right\| \leq \frac{(1-\zeta) t}{\zeta}\left(\left\|u_{t}-u\right\|+\left\|x_{t}-u\right\|\right) \rightarrow 0 \\
& \quad(\text { as } t \rightarrow 0) .
\end{aligned}
$$

Let $\left\{t_{n}\right\} \subset(0,1)$ be a sequence satisfying $t_{n} \rightarrow 0^{+}$as $n \rightarrow \infty$. Put $x_{n}:=x_{t_{n}}$. By (3.2), we get

$$
\lim _{n \rightarrow \infty}\left\|x_{n}-T x_{n}\right\|=0
$$

By (3.1), we obtain

$$
\begin{aligned}
\left\|x_{t}-p\right\|^{2} & =(1-\zeta)\left\langle\operatorname{proj}_{C}\left[t u_{t}+(1-t) x_{t}\right]-p, x_{t}-p\right\rangle+\zeta\left\langle T x_{t}-p, x_{t}-p\right\rangle \\
& \leq(1-\zeta)\left\|\operatorname{proj}_{C}\left[t u_{t}+(1-t) x_{t}\right]-p\right\|\left\|x_{t}-p\right\|+\zeta\left\|x_{t}-p\right\|^{2} \\
& \leq \frac{1-\zeta}{2}\left(\left\|\operatorname{proj}_{C}\left[t u_{t}+(1-t) x_{t}\right]-p\right\|^{2}+\left\|x_{t}-p\right\|^{2}\right)+\zeta\left\|x_{t}-p\right\|^{2} .
\end{aligned}
$$

Hence,

$$
\begin{aligned}
\left\|x_{t}-p\right\|^{2} & \leq\left\|\operatorname{proj}_{C}\left[t u_{t}+(1-t) x_{t}\right]-p\right\|^{2} \\
& \leq\left\|x_{t}-p+t\left(u_{t}-x_{t}\right)\right\|^{2}
\end{aligned}
$$




$$
\begin{aligned}
& =\left\|x_{t}-p\right\|^{2}+2 t\left\langle u_{t}-x_{t}, x_{t}-p\right\rangle+t^{2}\left\|u_{t}-x_{t}\right\|^{2} \\
& =\left\|x_{t}-p\right\|^{2}-2 t\left\langle x_{t}-p, x_{t}-p\right\rangle+2 t\left\langle u_{t}-p, x_{t}-p\right\rangle+t^{2}\left\|u_{t}-x_{t}\right\|^{2} \\
& =(1-2 t)\left\|x_{t}-p\right\|^{2}+2 t\left\langle u_{t}-p, x_{t}-p\right\rangle+t^{2}\left\|u_{t}-x_{t}\right\|^{2} .
\end{aligned}
$$

It follows that

$$
\begin{aligned}
\left\|x_{t}-p\right\|^{2} & \leq\left\langle u_{t}-p, x_{t}-p\right\rangle+\frac{t}{2}\left\|u_{t}-x_{t}\right\|^{2} \\
& \leq\left\langle u_{t}-p, x_{t}-p\right\rangle+t M_{1} .
\end{aligned}
$$

Here $M_{1}>0$ is a constant such that $\sup _{t \in(0,1)} \frac{\left\|u_{t}-x_{t}\right\|^{2}}{2} \leq M_{1}$. In particular, we obtain

$$
\left\|x_{n}-p\right\|^{2} \leq\left\langle u_{n}-p, x_{n}-p\right\rangle+t_{n} M_{1}, \quad \forall p \in \operatorname{Fix}(T) .
$$

Since $\left\{x_{n}\right\}$ is bounded, there exists a subsequence $\left\{x_{n_{i}}\right\}$ of $\left\{x_{n}\right\}$ satisfying $x_{n_{i}} \rightarrow x^{*} \in C$ weakly. By (3.3), we get

$$
\lim _{i \rightarrow \infty}\left\|x_{n_{i}}-T x_{n_{i}}\right\|=0
$$

Applying Lemma 2.1 to (3.6) to deduce $x^{*} \in \operatorname{Fix}(T)$.

By (3.5), we derive

$$
\left\|x_{n_{i}}-x^{*}\right\|^{2} \leq\left\langle u_{n_{i}}-x^{*}, x_{n_{i}}-x^{*}\right\rangle+t_{n_{i}} M_{1} .
$$

Since $u_{n_{i}}-x^{*} \rightarrow u-x^{*}$ and $t_{n_{i}} \rightarrow 0$, we deduce that $x_{n_{i}} \rightarrow x^{*}$ by (3.7). By (3.5), we have

$$
\left\|x^{*}-p\right\|^{2} \leq\left\langle u-p, x^{*}-p\right\rangle, \quad \forall p \in \operatorname{Fix}(T) .
$$

Assume that there exists another subsequence $\left\{x_{n_{j}}\right\}$ of $\left\{x_{n}\right\}$ satisfying $x_{n_{j}} \rightarrow x^{\dagger}$ weakly. Similarly, we can prove that $x_{n_{j}} \rightarrow x^{\dagger} \in \operatorname{Fix}(T)$, which satisfies

$$
\left\|x^{\dagger}-p\right\|^{2} \leq\left\langle u-p, x^{\dagger}-p\right\rangle, \quad \forall p \in \operatorname{Fix}(T) .
$$

In (3.8), we pick up $p=x^{\dagger}$ to get

$$
\left\|x^{*}-x^{\dagger}\right\|^{2} \leq\left\langle u-x^{\dagger}, x^{*}-x^{\dagger}\right\rangle
$$

In (3.9), we pick up $p=x^{*}$ to get

$$
\left\|x^{\dagger}-x^{*}\right\|^{2} \leq\left\langle u-x^{*}, x^{\dagger}-x^{*}\right\rangle
$$

Adding (3.10) and (3.11), we deduce

$$
\left\|x^{\dagger}-x^{*}\right\|^{2} \leq 0
$$

Thus, $x^{*}=x^{\dagger}$. This indicates that the weak limit set of $\left\{x_{n}\right\}$ is singleton and the path $\left\{x_{t}\right\}$ converges strongly to $x^{*}=\operatorname{proj}_{\mathrm{Fix}(T)}(u)$ by (3.8). This completes the proof. 
Corollary 3.3 The path $\left\{x_{t}\right\}$ defined by

$$
x_{t}=(1-\zeta) \operatorname{proj}_{C}\left[(1-t) x_{t}\right]+\zeta T x_{t}, \quad \forall t \in(0,1)
$$

converges strongly to $\operatorname{proj}_{\mathrm{Fix}(T)}(0)$, which is the minimum-norm fixed point of $T$.

Now, we introduce another algorithm, which is an explicit manner.

Algorithm 3.4 Let $\left\{\zeta_{n}\right\}$ and $\left\{\zeta_{n}\right\}$ be two real number sequences in $(0,1)$. Let $\left\{u_{n}\right\} \subset H$ be a sequence. For $x_{0} \in C$ arbitrarily, let the sequence $\left\{x_{n}\right\}$ be generated by

$$
x_{n+1}=\left(1-\zeta_{n}\right) \operatorname{proj}_{C}\left[\varsigma_{n} u_{n}+\left(1-\varsigma_{n}\right) x_{n}\right]+\zeta_{n} T x_{n}, \quad n \geq 0 .
$$

Theorem 3.5 Assume the following conditions are satisfied:

(C1) $\lim _{n \rightarrow \infty} \zeta_{n}=\lim _{n \rightarrow \infty} \frac{\zeta_{n}}{\zeta_{n}}=\lim _{n \rightarrow \infty} \frac{\zeta_{n}^{2}}{\zeta_{n}}=0$;

(C2) $\lim _{n \rightarrow \infty} u_{n}=u \in H$.

Then we have

(1) the sequence $\left\{x_{n}\right\}$ is bounded;

(2) the sequence $\left\{x_{n}\right\}$ is asymptotically regular, that is, $\lim _{n \rightarrow \infty}\left\|x_{n+1}-x_{n}\right\|=0$.

Further, if $\sum_{n=0}^{\infty} \varsigma_{n}=\infty$ and $\lim _{n \rightarrow \infty} \frac{\left\|x_{n+1}-x_{n}\right\|}{\zeta_{n}}=0$, then the sequence $\left\{x_{n}\right\}$ converges strongly to $\operatorname{proj}_{\mathrm{Fix}(T)}(u)$.

Proof By the condition (C1), we can find a sufficiently large positive integer $m$ such that

$$
1-\frac{1}{1 / 2-\zeta_{m}}(\kappa+1)(\kappa+2)\left(\zeta_{m}+2 \zeta_{m}+\frac{\zeta_{m}^{2}}{\zeta_{m}}\right)>0
$$

Let $p \in \operatorname{Fix}(T)$. For fixed $m$, we pick up a constant $M_{2}>0$ such that

$$
\max \left\{\left\|x_{0}-p\right\|,\left\|x_{1}-p\right\|, \ldots,\left\|x_{m-1}-p\right\|, 4\left\|x_{m}-p\right\|+4\left\|u_{m}-p\right\|\right\} \leq M_{2}
$$

Next, we show that $\left\|x_{m+1}-p\right\| \leq M_{2}$. Set $y_{n}=\operatorname{proj}_{C}\left[\varsigma_{n} u_{n}+\left(1-\varsigma_{n}\right) x_{n}\right]$ for all $n \geq 0$. Thus, we have $x_{n+1}=\left(1-\zeta_{n}\right) y_{n}+\zeta_{n} T x_{n}$ for all $n \geq 0$.

Since $I-T$ is monotone, we have

$$
\left\langle(I-T) x_{m+1}, x_{m+1}-p\right\rangle=\left\langle(I-T) x_{m+1}-(I-T) p, x_{m+1}-p\right\rangle \geq 0 .
$$

By (3.12), we obtain

$$
\begin{aligned}
\left\|x_{m+1}-p\right\|^{2}= & \left(1-\zeta_{m}\right)\left\langle y_{m}-p, x_{m+1}-p\right\rangle+\zeta_{m}\left\langle T x_{m}-p, x_{m+1}-p\right\rangle \\
= & \left(1-\zeta_{m}\right)\left\langle y_{m}-\zeta_{m} u_{m}-\left(1-\varsigma_{m}\right) x_{m}, x_{m+1}-p\right\rangle \\
& +\left(1-\zeta_{m}\right)\left\langle\zeta_{m} u_{m}+\left(1-\zeta_{m}\right) x_{m}-p, x_{m+1}-p\right\rangle \\
& +\zeta_{m}\left\langle T x_{m}-p, x_{m+1}-p\right\rangle \\
= & \left(1-\zeta_{m}\right)\left\langle y_{m}-\zeta_{m} u_{m}-\left(1-\zeta_{m}\right) x_{m}, x_{m+1}-p\right\rangle \\
& +\left(1-\zeta_{m}\right)\left\langle x_{m}-p, x_{m+1}-p\right\rangle+\left(1-\zeta_{m}\right) \zeta_{m}\left\langle u_{m}-x_{m}, x_{m+1}-p\right\rangle
\end{aligned}
$$




$$
\begin{aligned}
& +\zeta_{m}\left\langle T x_{m}-p, x_{m+1}-p\right\rangle \\
= & \left(1-\zeta_{m}\right)\left\langle y_{m}-\zeta_{m} u_{m}-\left(1-\zeta_{m}\right) x_{m}, x_{m+1}-p\right\rangle \\
& +\left\langle x_{m}-p, x_{m+1}-p\right\rangle-\left(1-\zeta_{m}\right) \zeta_{m}\left\langle x_{m+1}-p, x_{m+1}-p\right\rangle \\
& -\left(1-\zeta_{m}\right) \zeta_{m}\left\langle x_{m}-x_{m+1}, x_{m+1}-p\right\rangle-\left(1-\zeta_{m}\right) \varsigma_{m}\left\langle p-u_{m}, x_{m+1}-p\right\rangle \\
& +\zeta_{m}\left\langle T x_{m}-T x_{m+1}, x_{m+1}-p\right\rangle+\zeta_{m}\left\langle x_{m+1}-x_{m}, x_{m+1}-p\right\rangle \\
& -\zeta_{m}\left\langle x_{m+1}-T x_{m+1}, x_{m+1}-p\right\rangle .
\end{aligned}
$$

Note that

$$
\begin{aligned}
\left\|y_{m}-\varsigma_{m} u_{m}-\left(1-\varsigma_{m}\right) x_{m}\right\| & \leq\left\|y_{m}-x_{m}\right\|+\varsigma_{m}\left\|x_{m}-u_{m}\right\| \\
& =\left\|\operatorname{proj}_{C}\left[\varsigma_{m} u_{m}+\left(1-\varsigma_{m}\right) x_{m}\right]-x_{m}\right\|+\varsigma_{m}\left\|x_{m}-u_{m}\right\| \\
& \leq 2 \varsigma_{m}\left\|x_{m}-u_{m}\right\| .
\end{aligned}
$$

Then we have

$$
\begin{aligned}
\left\|x_{m+1}-p\right\|^{2} \leq & \left(1-\zeta_{m}\right)\left\|y_{m}-\zeta_{m} u_{m}-\left(1-\zeta_{m}\right) x_{m}\right\|\left\|x_{m+1}-p\right\| \\
& +\left\|x_{m}-p\right\|\left\|x_{m+1}-p\right\|-\left(1-\zeta_{m}\right) \zeta_{m}\left\|x_{m+1}-p\right\|^{2} \\
& +\left(1-\zeta_{m}\right) \zeta_{m}\left(\left\|x_{m+1}-x_{m}\right\|+\left\|u_{m}-p\right\|\right)\left\|x_{m+1}-p\right\| \\
& +\zeta_{m}\left(\left\|T x_{m}-T x_{m+1}\right\|+\left\|x_{m+1}-x_{m}\right\|\right)\left\|x_{m+1}-p\right\| \\
\leq & 2\left(1-\zeta_{m}\right) \zeta_{m}\left\|x_{m}-u_{m}\right\|\left\|x_{m+1}-p\right\|+\left\|x_{m}-p\right\|\left\|x_{m+1}-p\right\| \\
& +\left(1-\zeta_{m}\right) \zeta_{m}\left(\left\|x_{m+1}-x_{m}\right\|+\left\|u_{m}-p\right\|\right)\left\|x_{m+1}-p\right\| \\
& -\left(1-\zeta_{m}\right) \zeta_{m}\left\|x_{m+1}-p\right\|^{2}+\zeta_{m}(\kappa+1)\left\|x_{m+1}-x_{m}\right\|\left\|x_{m+1}-p\right\| \\
\leq & \left\|x_{m}-p\right\|\left\|x_{m+1}-p\right\|+2\left(1-\zeta_{m}\right) \zeta_{m}\left(\left\|x_{m}-p\right\|+\left\|u_{m}-p\right\|\right)\left\|x_{m+1}-p\right\| \\
& -\left(1-\zeta_{m}\right) \zeta_{m}\left\|x_{m+1}-p\right\|^{2}+\left(\zeta_{m}+\zeta_{m}\right)(\kappa+1)\left\|x_{m+1}-x_{m}\right\|\left\|x_{m+1}-p\right\| .
\end{aligned}
$$

Hence,

$$
\begin{aligned}
& {\left[1+\left(1-\zeta_{m}\right) \varsigma_{m}\right]\left\|x_{m+1}-p\right\| \leq\left\|x_{m}-p\right\|+2 \varsigma_{m}\left(\left\|x_{m}-p\right\|+\left\|u_{m}-p\right\|\right)} \\
& +(\kappa+1)\left(\varsigma_{m}+\zeta_{m}\right)\left\|x_{m+1}-x_{m}\right\| \text {. }
\end{aligned}
$$

By (3.12), we have

$$
\begin{aligned}
\left\|x_{m+1}-x_{m}\right\| & \leq\left(1-\zeta_{m}\right)\left\|\operatorname{proj}_{C}\left[\zeta_{m} u_{m}+\left(1-\zeta_{m}\right) x_{m}\right]-x_{m}\right\|+\zeta_{m}\left\|T x_{m}-x_{m}\right\| \\
& \leq\left(1-\zeta_{m}\right) \varsigma_{m}\left(\left\|x_{m}-p\right\|+\left\|u_{m}-p\right\|\right)+\zeta_{m}\left(\left\|T x_{m}-p\right\|+\left\|p-x_{m}\right\|\right) \\
& \leq \varsigma_{m}\left(\left\|x_{m}-p\right\|+\left\|u_{m}-p\right\|\right)+\zeta_{m}(\kappa+1)\left\|x_{m}-p\right\| \\
& \leq(\kappa+1)\left(\zeta_{m}+\zeta_{m}\right)\left\|x_{m}-p\right\|+\zeta_{m}\left\|u_{m}-p\right\| \\
& \leq(\kappa+2)\left(\zeta_{m}+\zeta_{m}\right) M_{2} .
\end{aligned}
$$


From condition (C1), we deduce $\zeta_{m} \rightarrow 0$ and $\zeta_{m} \rightarrow 0$ as $m \rightarrow \infty$. Therefore, we get

$$
\lim _{m \rightarrow \infty}\left\|x_{m+1}-x_{m}\right\|=0
$$

That is, the sequence $\left\{x_{m}\right\}$ is asymptotically regular.

By (3.15) and (3.16), we have

$$
\begin{aligned}
{[1} & \left.+\left(1-\zeta_{m}\right) \zeta_{m}\right]\left\|x_{m+1}-p\right\| \\
& \leq\left\|x_{m}-p\right\|+\varsigma_{m}\left(2\left\|x_{m}-p\right\|+2\left\|u_{m}-p\right\|\right)+(\kappa+1)(\kappa+2)\left(\varsigma_{m}+\zeta_{m}\right)^{2} M_{2} \\
& \leq\left(1+\frac{1}{2} \varsigma_{m}\right) M_{2}+(\kappa+1)(\kappa+2)\left(\varsigma_{m}+\zeta_{m}\right)^{2} M_{2} .
\end{aligned}
$$

This together with (3.13) and (3.14) imply that

$$
\begin{aligned}
\left\|x_{m+1}-p\right\| & \leq\left[1-\frac{\left(1 / 2-\zeta_{m}\right) \zeta_{m}-(\kappa+1)(\kappa+2)\left(\zeta_{m}+\zeta_{m}\right)^{2}}{1+\left(1-\zeta_{m}\right) \zeta_{m}}\right] M_{2} \\
& =\left\{1-\frac{\left(1 / 2-\zeta_{m}\right) \zeta_{m}\left[1-\frac{1}{1 / 2-\zeta_{m}}(\kappa+1)(\kappa+2)\left(\zeta_{m}+2 \zeta_{m}+\left(\zeta_{m}^{2} / \zeta_{m}\right)\right)\right]}{1+\left(1-\zeta_{m}\right) \zeta_{m}}\right\} M_{2} \\
& \leq M_{2} .
\end{aligned}
$$

By induction, we get

$$
\left\|x_{n}-p\right\| \leq M_{2}, \quad \forall n \geq 0
$$

So $\left\{x_{n}\right\}$ is bounded.

By (3.12), we have

$$
\begin{aligned}
\left\|x_{n}-T x_{n}\right\| & \leq\left\|x_{n}-x_{n+1}\right\|+\left\|x_{n+1}-T x_{n}\right\| \\
& \leq\left\|x_{n}-x_{n+1}\right\|+\left(1-\zeta_{n}\right)\left\|\operatorname{proj}_{C}\left[\varsigma_{n} u_{n}+\left(1-\varsigma_{n}\right) x_{n}\right]-T x_{n}\right\| \\
& \leq\left\|x_{n}-x_{n+1}\right\|+\left(1-\zeta_{n}\right)\left\|x_{n}-T x_{n}\right\|+\varsigma_{n}\left\|x_{n}-u_{n}\right\| .
\end{aligned}
$$

It follows that

$$
\left\|x_{n}-T x_{n}\right\| \leq \frac{1}{\zeta_{n}}\left\|x_{n}-x_{n+1}\right\|+\frac{\zeta_{n}}{\zeta_{n}}\left\|x_{n}-u_{n}\right\|
$$

By the condition $\lim _{n \rightarrow \infty} \frac{\zeta_{n}}{\zeta_{n}}=0$ and the assumption $\lim _{n \rightarrow \infty} \frac{\left\|x_{n+1}-x_{n}\right\|}{\zeta_{n}}=0$, we deduce

$$
\lim _{n \rightarrow \infty}\left\|x_{n}-T x_{n}\right\|=0
$$

Let the net $\left\{z_{t}\right\}$ be defined by $z_{t}=(1-\zeta) \operatorname{proj}_{C}\left[t u_{t}+(1-t) z_{t}\right]+\zeta T z_{t}$. By Theorem 3.2, we know that $z_{t}$ converges strongly to $\operatorname{proj}_{\mathrm{Fix}(T)}(u)$. Next, we prove

$$
\limsup _{n \rightarrow \infty}\left\langle\operatorname{proj}_{\mathrm{Fix}(T)}(u)-u_{n}, \operatorname{proj}_{\mathrm{Fix}(T)}(u)-y_{n}\right\rangle \leq 0 .
$$


By the definition of $\left\{z_{t}\right\}$, we have

$$
z_{t}-x_{n}=(1-\zeta)\left(\operatorname{proj}_{C}\left[t u_{t}+(1-t) z_{t}\right]-x_{n}\right)+\zeta\left(T z_{t}-T x_{n}\right)+\zeta\left(T x_{n}-x_{n}\right)
$$

It follows that

$$
\begin{aligned}
\left\|z_{t}-x_{n}\right\|^{2}= & (1-\zeta)\left\langle\operatorname{proj}_{C}\left[t u_{t}+(1-t) z_{t}\right]-x_{n}, z_{t}-x_{n}\right\rangle+\zeta\left\langle T z_{t}-T x_{n}, z_{t}-x_{n}\right\rangle \\
& +\zeta\left\langle T x_{n}-x_{n}, z_{t}-x_{n}\right\rangle \\
= & (1-\zeta)\left\langle\operatorname{proj}_{C}\left[t u_{t}+(1-t) z_{t}\right]-t u_{t}-(1-t) z_{t}, z_{t}-x_{n}\right\rangle \\
& +(1-\zeta)\left\langle t u_{t}+(1-t) z_{t}-x_{n}, z_{t}-x_{n}\right\rangle+\zeta\left\langle T z_{t}-T x_{n}, z_{t}-x_{n}\right\rangle \\
& +\zeta\left\langle T x_{n}-x_{n}, z_{t}-x_{n}\right\rangle .
\end{aligned}
$$

Since $x_{n} \in C$, by the characteristic inequality of metric projection, we have

$$
\left\langle\operatorname{proj}_{C}\left[t u_{t}+(1-t) z_{t}\right]-t u_{t}-(1-t) z_{t}, z_{t}-x_{n}\right\rangle \leq 0
$$

Then

$$
\begin{aligned}
\left\|z_{t}-x_{n}\right\|^{2} \leq & (1-\zeta)\left\langle t u_{t}+(1-t) z_{t}-x_{n}, z_{t}-x_{n}\right\rangle+\zeta\left\|z_{t}-x_{n}\right\|^{2} \\
& +\zeta\left\|T x_{n}-x_{n}\right\|\left\|z_{t}-x_{n}\right\| \\
= & (1-\zeta)\left\|z_{t}-x_{n}\right\|^{2}-(1-\zeta) t\left\langle z_{t}-u_{t}, z_{t}-x_{n}\right\rangle+\zeta\left\|z_{t}-x_{n}\right\|^{2} \\
& +\zeta\left\|T x_{n}-x_{n}\right\|\left\|z_{t}-x_{n}\right\|,
\end{aligned}
$$

which implies that

$$
\left\langle z_{t}-u_{t}, z_{t}-x_{n}\right\rangle \leq \frac{\zeta}{(1-\zeta) t}\left\|T x_{n}-x_{n}\right\|\left\|z_{t}-x_{n}\right\|
$$

By (3.17), we deduce

$$
\limsup _{t \rightarrow 0} \limsup _{n \rightarrow \infty}\left\langle z_{t}-u_{t}, z_{t}-x_{n}\right\rangle \leq 0
$$

Note the fact that the two limits $\limsup _{t \rightarrow 0}$ and $\lim \sup _{n \rightarrow \infty}$ are interchangeable. This together with $z_{t} \rightarrow \operatorname{proj}_{\mathrm{Fix}(T)}(u), u_{t} \rightarrow u$ and (3.18) implies that

$$
\limsup _{n \rightarrow \infty}\left\langle\operatorname{proj}_{\text {Fix }(T)}(u)-u, \operatorname{proj}_{\text {Fix }(T)}(u)-x_{n}\right\rangle \leq 0 \text {. }
$$

Note that $\left\|y_{n}-x_{n}\right\| \rightarrow 0$ and $u_{n}-u \rightarrow 0$. We derive

$$
\limsup _{n \rightarrow \infty}\left\langle\operatorname{proj}_{\mathrm{Fix}(T)}(u)-u_{n}, \operatorname{proj}_{\mathrm{Fix}(T)}(u)-y_{n}\right\rangle \leq 0 .
$$


Finally, we prove that $x_{n} \rightarrow \operatorname{proj}_{\text {Fix }(T)}(u)$. Note that

$$
\begin{aligned}
& \left\langle T x_{n}-\operatorname{proj}_{\mathrm{Fix}(T)}(u), x_{n+1}-\operatorname{proj}_{\mathrm{Fix}(T)}(u)\right\rangle \\
& \quad=\left\langle T x_{n}-\operatorname{proj}_{\mathrm{Fix}(T)}(u), x_{n}-\operatorname{proj}_{\mathrm{Fix}(T)}(u)\right\rangle+\left\langle T x_{n}-\operatorname{proj}_{\mathrm{Fix}(T)}(u), x_{n+1}-x_{n}\right\rangle \\
& \quad \leq\left\|x_{n}-\operatorname{proj}_{\mathrm{Fix}(T)}(u)\right\|^{2}+\left\|T x_{n}-\operatorname{proj}_{\mathrm{Fix}(T)}(u)\right\|\left\|x_{n+1}-x_{n}\right\|
\end{aligned}
$$

and

$$
\begin{aligned}
\| y_{n}- & \operatorname{proj}_{\mathrm{Fix}(T)}(u) \|^{2} \\
= & \left\langle y_{n}-\varsigma_{n} u_{n}-\left(1-\varsigma_{n}\right) x_{n}, y_{n}-\operatorname{proj}_{\mathrm{Fix}(T)}(u)\right\rangle \\
& +\left\langle\zeta_{n} u_{n}+\left(1-\varsigma_{n}\right) x_{n}-\operatorname{proj}_{\mathrm{Fix}(T)}(u), y_{n}-\operatorname{proj}_{\mathrm{Fix}(T)}(u)\right\rangle \\
\leq & \left\langle\zeta_{n} u_{n}+\left(1-\varsigma_{n}\right) x_{n}-\operatorname{proj}_{\mathrm{Fix}(T)}(u), y_{n}-\operatorname{proj}_{\mathrm{Fix}(T)}(u)\right\rangle \\
= & \left(1-\varsigma_{n}\right)\left\langle x_{n}-\operatorname{proj}_{\mathrm{Fix}(T)}(u), y_{n}-\operatorname{proj}_{\mathrm{Fix}(T)}(u)\right\rangle \\
& -\varsigma_{n}\left\langle\operatorname{proj}_{\mathrm{Fix}(T)}(u)-u_{n}, y_{n}-\operatorname{proj}_{\mathrm{Fix}(T)}(u)\right\rangle \\
\leq & \frac{\left(1-\varsigma_{n}\right)}{2}\left\|x_{n}-\operatorname{proj}_{\mathrm{Fix}(T)}(u)\right\|^{2}+\frac{1}{2}\left\|y_{n}-\operatorname{proj}_{\mathrm{Fix}(T)}(u)\right\|^{2} \\
& -\varsigma_{n}\left\langle\operatorname{proj}_{\mathrm{Fix}(T)}(u)-u_{n}, y_{n}-\operatorname{proj}_{\mathrm{Fix}(T)}(u)\right\rangle .
\end{aligned}
$$

Then

$$
\begin{aligned}
\left\|y_{n}-\operatorname{proj}_{\text {Fix }(T)}(u)\right\|^{2} \leq & \left(1-\varsigma_{n}\right)\left\|x_{n}-\operatorname{proj}_{\text {Fix }(T)}(u)\right\|^{2} \\
& -2 \varsigma_{n}\left\langle\operatorname{proj}_{\text {Fix }(T)}(u)-u_{n}, y_{n}-\operatorname{proj}_{\text {Fix }(T)}(u)\right\rangle .
\end{aligned}
$$

By (3.12), (3.16), and (3.20), we get

$$
\begin{aligned}
\| x_{n+1} & -\operatorname{proj}_{\mathrm{Fix}(T)}(u) \|^{2} \\
= & \left\|\left(1-\zeta_{n}\right)\left(y_{n}-\operatorname{proj}_{\mathrm{Fix}(T)}(u)\right)+\zeta_{n}\left(T x_{n}-\operatorname{proj}_{\mathrm{Fix}(T)}(u)\right)\right\|^{2} \\
\leq & \left\|\left(1-\zeta_{n}\right)\left(y_{n}-\operatorname{proj}_{\mathrm{Fix}(T)}(u)\right)\right\|^{2} \\
& +2 \zeta_{n}\left\langle T x_{n}-\operatorname{proj}_{\mathrm{Fix}(T)}(u), x_{n+1}-\operatorname{proj}_{\mathrm{Fix}(T)}(u)\right\rangle \\
\leq & \left(1-\zeta_{n}\right)^{2}\left(1-\zeta_{n}\right)\left\|x_{n}-\operatorname{proj}_{\mathrm{Fix}(T)}(u)\right\|^{2}+2 \zeta_{n}\left\|x_{n}-\operatorname{proj}_{\mathrm{Fix}(T)}(u)\right\|^{2} \\
& -2 \zeta_{n}\left(1-\zeta_{n}\right)^{2}\left\langle\operatorname{proj}_{\mathrm{Fix}(T)}(u)-u_{n}, y_{n}-\operatorname{proj}_{\mathrm{Fix}(T)}(u)\right\rangle \\
& +2 \zeta_{n}\left\|T x_{n}-\operatorname{proj}_{\mathrm{Fix}(T)}(u)\right\|\left\|x_{n+1}-x_{n}\right\| \\
\leq & {\left[1-\left(1-2 \zeta_{n}\right) \zeta_{n}\right]\left\|x_{n}-\operatorname{proj}_{\mathrm{Fix}(T)}(u)\right\|^{2}+\zeta_{n}^{2}\left\|x_{n}-\operatorname{proj}_{\mathrm{Fix}(T)}(u)\right\|^{2} } \\
& +2 \zeta_{n}\left(1-\zeta_{n}\right)^{2}\left\langle\operatorname{proj}_{\mathrm{Fix}(T)}(u)-u_{n}, \operatorname{proj}_{\mathrm{Fix}(T)}(u)-y_{n}\right\rangle \\
& +2 \zeta_{n}\left\|T x_{n}-\operatorname{proj}_{\mathrm{Fix}(T)}(u)\right\|(\kappa+2)\left(\zeta_{n}+\zeta_{n}\right) M_{2} \\
= & \left(1-\gamma_{n}\right)\left\|x_{n}-\operatorname{proj}_{\mathrm{Fix}(T)}(u)\right\|^{2}+\gamma_{n} \delta_{n},
\end{aligned}
$$


where $\gamma_{n}=\left(1-2 \zeta_{n}\right) \varsigma_{n}$ and

$$
\begin{aligned}
\delta_{n}= & \frac{2\left(1-\zeta_{n}\right)^{2}}{1-2 \zeta_{n}}\left\langle\operatorname{proj}_{\operatorname{Fix}(T)}(u)-u_{n}, \operatorname{proj}_{\mathrm{Fix}(T)}(u)-y_{n}\right\rangle+\frac{\zeta_{n}^{2}}{\left(1-2 \zeta_{n}\right) \zeta_{n}}\left\|x_{n}-\operatorname{proj}_{\operatorname{Fix}(T)}(u)\right\|^{2} \\
& +\frac{2 \zeta_{n}}{1-2 \zeta_{n}}\left\|T x_{n}-\operatorname{proj}_{\mathrm{Fix}(T)}(u)\right\|(\kappa+2) M_{2} \\
& +\frac{2 \zeta_{n}^{2}}{\left(1-2 \zeta_{n}\right) \zeta_{n}}\left\|T x_{n}-\operatorname{proj}_{\mathrm{Fix}(T)}(u)\right\|(\kappa+2) M_{2} .
\end{aligned}
$$

It is clear that $\sum_{n=0}^{\infty} \gamma_{n}=\infty$ and $\limsup _{n \rightarrow \infty} \delta_{n} \leq 0$. We can therefore apply Lemma 2.2 to (3.21) and conclude that $x_{n} \rightarrow \operatorname{proj}_{\text {Fix }(T)}(u)$ as $n \rightarrow \infty$. This completes the proof.

Corollary 3.6 Let $\left\{\zeta_{n}\right\}$ and $\left\{\zeta_{n}\right\}$ be two real number sequences in $(0,1)$. For $x_{0} \in C$ arbitrarily, let the sequence $\left\{x_{n}\right\}$ be generated by

$$
x_{n+1}=\left(1-\zeta_{n}\right) \operatorname{proj}_{C}\left[\left(1-\zeta_{n}\right) x_{n}\right]+\zeta_{n} T x_{n}, \quad n \geq 0 .
$$

Assume $\lim _{n \rightarrow \infty} \zeta_{n}=\lim _{n \rightarrow \infty} \frac{\zeta_{n}}{\zeta_{n}}=\lim _{n \rightarrow \infty} \frac{\zeta_{n}^{2}}{\zeta_{n}}=0$. Then we have

(1) the sequence $\left\{x_{n}\right\}$ is bounded;

(2) the sequence $\left\{x_{n}\right\}$ is asymptotically regular, that is, $\lim _{n \rightarrow \infty}\left\|x_{n+1}-x_{n}\right\|=0$.

Further, if $\sum_{n=0}^{\infty} \varsigma_{n}=\infty$ and $\lim _{n \rightarrow \infty} \frac{\left\|x_{n+1}-x_{n}\right\|}{\zeta_{n}}=0$, then the sequence $\left\{x_{n}\right\}$ converges strongly to $\operatorname{proj}_{\text {Fix }(T)}(0)$, which is the minimum-norm fixed point of $T$.

Proof Letting $u_{n}=u=0$ in (3.12), we obtain (3.22). Consequently, by Theorem 3.5, we find that the sequence $\left\{x_{n}\right\}$ generated by (3.22) converges strongly to $\operatorname{proj}_{\operatorname{Fix}(T)}(0)$, which is the minimum-norm fixed point of $T$.

\section{Competing interests}

The authors declare that they have no competing interests.

\section{Authors' contributions}

All authors read and approved the final manuscript.

\section{Author details}

${ }^{1}$ School of Electrical Engineering and Automation, Tianjin Polytechnic University, Tianjin, 300387, China. ${ }^{2}$ Department of Mathematics and RINS, Gyeongsang National University, Jinju, 660-701, Korea. ${ }^{3}$ Department of Business Administration, Gyeongsang National University, Jinju, 660-701, Korea.

\section{Acknowledgements}

The authors are very grateful to the reviewers for their valuable comments and suggestions.

Received: 15 March 2014 Accepted: 15 September 2014 Published: 9 October 2014

\section{References}

1. Ceng, LC, Petruşel, A, Yao, JC: Strong convergence of modified implicit iterative algorithms with perturbed mappings for continuous pseudocontractive mappings. Appl. Math. Comput. 209, 162-176 (2009). doi:10.1016/j.amc.2008.10.062

2. Chidume, CE, Mutangadura, SA: An example on the Mann iteration method for Lipschitz pseudo-contractions. Proc Am. Math. Soc. 129, 2359-2363 (2001). doi:10.1090/S0002-9939-01-06009-9

3. Chidume, $\mathrm{CE}$, Zegeye, $\mathrm{H}$ : Approximate fixed point sequences and convergence theorems for Lipschitz pseudo-contractive maps. Proc. Am. Math. Soc. 132, 831-840 (2004). doi:10.1090/S0002-9939-03-07101-6

4. Cho, SY, Qin, X, Kang, SM: Hybrid projection algorithms for treating common fixed points of a family of demicontinuous pseudocontractions. Appl. Math. Lett. 25, 854-857 (2012). doi:10.1016/j.aml.2011.10.031

5. Ishikawa, S: Fixed points by a new iteration method. Proc. Am. Math. Soc. 44, 147-150 (1974). doi:10.1090/S0002-9939-1974-0336469-5 
6. Kang, SM, Cho, SY, Qin, X: Hybrid projection algorithms for approximating fixed points of asymptotically quasi-pseudocontractive mappings. J. Nonlinear Sci. Appl. 5, 466-474 (2012)

7. Kang, SM, Rafiq, A: On convergence results for Lipschitz pseudocontractive mappings. J. Appl. Math. 2012, Article ID 902601 (2012). doi:10.1155/2012/902601

8. Kang, SM, Rafiq, A, Lee, S: Convergence analysis of an iterative scheme for Lipschitzian hemicontractive mappings in Hilbert spaces. J. Inequal. Appl. 2013, Article ID 132 (2013). doi:10.1186/1029-242X-2013-132

9. Li, XS, Kim, JK, Huang, NJ: Viscosity approximation of common fixed points for L-Lipschitzian semigroup of pseudocontractive mappings in Banach spaces. J. Inequal. Appl. 2009, Article ID 936121 (2009). doi:10.1155/2009/936121

10. Mann, WR: Mean value methods in iteration. Proc. Am. Math. Soc. 4, 506-510 (1953). doi:10.1090/S0002-9939-1953-0054846-3

11. Morales, $\mathrm{CH}$, Jung, JS: Convergence of paths for pseudocontractive mappings in Banach spaces. Proc. Am. Math. Soc. 128, 3411-3419 (2000). doi:10.1090/S0002-9939-00-05573-8

12. Ofoedu, EU, Zegeye, H: Further investigation on iteration processes for pseudocontractive mappings with application. Nonlinear Anal. 75, 153-162 (2012). doi:10.1016/j.na.2011.08.015

13. Qin, X, Cho, YJ, Kang, SM, Zhou, H: Convergence theorems of common fixed points for a family of Lipschitz quasi-pseudocontractions. Nonlinear Anal. 71, 685-690 (2009). doi:10.1016/j.na.2008.10.102

14. Song, YS, Chen, R: An approximation method for continuous pseudocontractive mappings. J. Inequal. Appl. 2006, Article ID 28950 (2006). doi:10.1155/JIA/2006/28950

15. Udomene, A: Path convergence, approximation of fixed points and variational solutions of Lipschitz pseudocontractions in Banach spaces. Nonlinear Anal. 67, 2403-2414 (2007). doi:10.1016/j.na.2006.09.001

16. Wen, DJ, Chen, YA: General iterative method for generalized equilibrium problems and fixed point problems of k-strict pseudo-contractions. Fixed Point Theory Appl. 2012, Article ID 125 (2012). doi:10.1186/1687-1812-2012-125

17. Yao, Y, Colao, V, Marino, G, Xu, HK: Implicit and explicit algorithms for minimum-norm fixed points of pseudocontractions in Hilbert spaces. Taiwan. J. Math. 16, 1489-1506 (2012)

18. Yao, Y, Liou, YC: Strong convergence of an implicit iteration algorithm for a finite family of pseudocontractive mappings. J. Inequal. Appl. 2008, Article ID 280908 (2008). doi:10.1155/2008/280908

19. Yao, Y, Liou, YC, Chen, R: Strong convergence of an iterative algorithm for pseudocontractive mapping in Banach spaces. Nonlinear Anal. 67, 3311-3317 (2007). doi:10.1016/j.na.2006.10.013

20. Yao, Y, Liou, YC, Marino, G: A hybrid algorithm for pseudo-contractive mappings. Nonlinear Anal. 71, 4997-5002 (2009). doi:10.1016/j.na.2009.03.075

21. Yao, Y, Marino, G, Xu, HK, Liou, YC: Construction of minimum-norm fixed points of pseudocontractions in Hilbert spaces. J. Inequal. Appl. 2014, Article ID 206 (2014). doi:10.1186/1029-242X-2014-206

22. Zegeye, H, Shahzad, N, Alghamdi, MA: Convergence of Ishikawa's iteration method for pseudocontractive mappings. Nonlinear Anal. 74, 7304-7311 (2011). doi:10.1016/j.na.2011.07.048

23. Zegeye, H, Shahzad, N, Alghamdi, MA: Minimum-norm fixed point of pseudocontractive mappings. Abstr. Appl. Anal. 2012, Article ID 926017 (2012). doi:10.1155/2012/926017

24. Zhou, $\mathrm{H}$ : Strong convergence of an explicit iterative algorithm for continuous pseudo-contractions in Banach spaces. Nonlinear Anal. 70, 4039-4046 (2009). doi:10.1016/j.na.2008.08.012

25. Yao, Y, Shahzad, N: New methods with perturbations for non-expansive mappings in Hilbert spaces. Fixed Point Theory Appl. 2011, Article ID 79 (2011). doi:10.1186/1687-1812-2011-79

26. Yu, ZT, Lin, $\sqcup$, Chuang, CS: Mathematical programming with multiple sets split monotone variational inclusion constraints. Fixed Point Theory Appl. 2014, Article ID 20 (2014). doi:10.1186/1687-1812-2014-20

27. Xu, HK: Iterative algorithms for nonlinear operators. J. Lond. Math. Soc. 66, 240-256 (2002). doi:10.1112/s0024610702003332

doi:10.1186/1029-242X-2014-386

Cite this article as: Guo et al.: Algorithms with variant anchors for pseudocontractive mappings. Journal of Inequalities and Applications 2014 2014:386.

\section{Submit your manuscript to a SpringerOpen ${ }^{\ominus}$ journal and benefit from:}

- Convenient online submission

- Rigorous peer review

- Immediate publication on acceptance

- Open access: articles freely available online

- High visibility within the field

- Retaining the copyright to your article

Submit your next manuscript at $>$ springeropen.com 